\title{
Um Guia didático para docentes: em busca dos logaritmos na comparação entre termos de diferentes sequências
}

\author{
A didactic guide for teachers: searching for the logarithms in the comparison \\ between terms of different sequences
}

\author{
Daniela Mendes Vieira da Silva * \\ Dora Soraia Kindel *
}

\section{Resumo}

O presente guia didático se constitui no produto resultante de uma dissertação de Mestrado que discutiu produções escritas de estudantes do Ensino Médio acerca de propriedades de logaritmos em um ambiente investigativo. Para o desenvolvimento deste trabalho apoiamo-nos no Experimento de design. Dentro deste aporte teórico fizemos a fundamentação Teórico Metodológica desta pesquisa e estabelecemos o quadro de ensino aprendizagem de logaritmos a partir da análise de um livro de referência no tema para a formação de professores, duas coleções de livros didáticos adotadas pela escola que sedia esta pesquisa e dissertações sobre o ensino do tema e um estudo conceitual do objeto matemático logaritmo. Em um segundo momento, utilizamos os cenários para investigação aliados à investigação em sala de aula para elaborar um conjunto de tarefas que facilitasse a introdução do tema logaritmos. Conjunto esse que testamos e reelaboramos em um ciclo de aplicações e aplicamos como instrumento de coleta das produções textuais dos participantes da pesquisa em um segundo ciclo. Os dados analisados com o apoio da Teoria dos Registros de Representação Semiótica - TRRS indicaram 0 alcance de diversas propriedades dos logaritmos a partir das tarefas propostas aos participantes assim como a adesão dos mesmos à investigação proposta.

Palavras-chave: Educação Matemática. Aprendizagem de logaritmos. Guia didático para professores.

\begin{abstract}
This didactic guide is the product of a Master's dissertation that discussed written productions of high school students about logarithmic properties in a research environment. For the development of this work we rely on the Design Experiment. Within this theoretical contribution we made the Theoretical Methodological basis of this research and established the learning framework of logarithms from the analysis of a reference book on the theme for teacher training, two collections of textbooks adopted by the school

\footnotetext{
* Doutoranda em Ensino de Matemática PEMAT/UFRJ, Docente das licenciaturas em Matemática e Pedagogia e da Pós Graduação Lato Sensu em Ensino de Matemática da Universidade Castelo Branco.

* Docente das licenciaturas em Matemática e Pedagogia da UFRRJ e do Programa de Pós Graduação Strictu Sensu em Educação em Ciências e Matemática.
} 
that hosts this research and dissertations on the teaching of the subject and a conceptual study of the logarithm mathematical object. Second, we used the research scenarios associated with classroom research to elaborate a set of tasks that facilitated the introduction of the logarithms theme. This set we tested and reworked in a cycle of applications and applied as an instrument to collect the textual productions of the research participants in a second cycle. The data analyzed with the support of Theory of Registers of Semiotic Representation - TRRS indicated the reach of several properties of the logarithms from the tasks proposed to the participants as well as their adherence to the proposed research.

Keywords: Mathematical Education; Learning of logarithms; Teaching guide for teachers.

\section{Introdução}

O tema em foco surge a partir das necessidades curriculares da instituição que abriga a pesquisa que originou este guia e na qual atuo como professora. Trata-se de uma escola técnica em telecomunicações em que há uma demanda do corpo técnico da escola por um trabalho bem fundamentado na formação do conceito de logaritmo, uma vez que todas as unidades de medidas utilizadas em telecomunicações são derivadas dos logaritmos nas bases binária, neperiana e decimal.

As áreas de análise e projetos de sistemas e medidas em telecomunicações requerem o uso intensivo do decibel ${ }^{1}$. Algumas das equações, tais como o teorema de Shanon, que limita a taxa máxima de um canal e atenuação em espaço livre são calculadas por logaritmos respectivamente binário e decimal. A própria teoria da informação, base das telecomunicações é definida por um logaritmo na base binária. O logaritmo está permeado em todas as disciplinas do curso técnico em telecomunicações ${ }^{2} e$, principalmente, nas disciplinas sistemas de telecomunicações, redes de fibra ótica, sistemas de satélite, infraestrutura. (NAVAS, 2015).

Para que a aprendizagem dos estudantes e não o conteúdo esteja em primeiro plano, uma vez que o estudo de logaritmos é uma necessidade do currículo técnico dos estudantes pesquisados buscamos o distanciamento do Ensino Tradicional Vigente - ETV a respeito do qual sabemos que:

\footnotetext{
${ }^{1}$ Definido a partir do logaritmo na base 10 .

${ }^{2}$ Oferecido pela escola que abriga esta pesquisa.
} 
[...] no ensino tradicional vigente, aqui denominado ETV, o aluno se mantém numa situação de "fazer de conta" que está entendendo, para que o professor possa chegar mais rapidamente, sem digressões ou interrupções, à solução "oficial". O aluno, ao invés de procurar descobrir se estaria em condições de oferecer uma solução possível, concentra-se em "adivinhar" a que solução o professor pretende chegar ou qual resposta quer ouvir. Não há lugar para o significado, mas apenas para uma negociação mútua a respeito do que se supõe que deva ser entendido (SILVA, 1998, p.1).

O ETV se enquadra no paradigma do exercício, ou seja, numa prática excessiva de tarefas cuja resposta é única e já sabida, em que se espera do aluno uma solução desta tarefa, inclusive, muitas vezes percorrendo até mesmo um caminho pré-determinado até ela. Esse paradigma se diferencia do cenário para investigação que se apresentou como uma opção para o rompimento com o mesmo, no qual os estudantes são convidados a se envolverem em processos de exploração e argumentação justificada.

Observamos que fazer a movimentação do paradigma do exercício em direção ao cenário para investigação se constituiu em um auxiliar para o enfraquecimento da autoridade da sala de aula nos moldes do ETV e a engajar os estudantes ativamente em seus processos de aprendizagem. Entendemos, portanto, que caminhar entre os diferentes ambientes de aprendizagem pode ser uma forma de engajar os estudantes em ação e reflexão. Postura desejada por nós neste trabalho (SKOVSMOSE, 2000).

\section{OBJETIVO E ESTRUTURA DO GUIA}

Nosso objetivo com este guia didático é o de compartilhar com outros docentes os frutos de nossa pesquisa que discute uma introdução ao conceito de logaritmos por investigação através da escrita e da fala dos estudantes participantes. Pretendemos aqui apresentar aos professores um conjunto de tarefas para a introdução ao conceito de logaritmos como operações entre expoentes. O conjunto que propomos aqui propicia a coordenação de representações, uma vez que, segundo Duval (2009) quanto mais representações o aprendente tiver oportunidade de coordenar, melhor ele se acercará do conceito matemático. Além disto, buscamos estimular coordenações cognitivamente 
econômicas nesta tarefa, uma vez que estão relacionadas com índices de sucesso escolar (ibid).

A primeira unidade, do presente trabalho, apresenta a intencionalidade do presente guia e sua estrutura. $\mathrm{Na}$ segunda unidade apresentamos a fundamentação teórica a partir da qual elaboramos o conjunto de tarefas que apresentaremos mais adiante. A terceira unidade apresenta os logaritmos como operações entre expoentes preparando a compreensão da unidade 4 na qual o conjunto de tarefas é apresentado detalhadamente neste guia. A quarta unidade traz o conjunto de tarefas para a introdução ao conceito de logaritmos por investigação dentro da TRRS. Finalizamos este guia com a bibliografia consultada para a confecção deste trabalho e no apêndice disponibilizamos o conjunto de tarefas completo sobre o qual discorremos na unidade 4 em uma versão para impressão.

\section{FUNDAMENTAÇÃO TEÓRICA}

A TRRS dá suporte a diversos estudos que visam compreender a forma como as pessoas constroem o conhecimento matemático. Esta teoria tem relação com a representação, tratamento e conversão de conceitos matemáticos.

Dentro da TRRS, o foco deve estar no aprendiz, o que subordina o objeto a ser ensinado à cognição deste, cognição esta que se liga às questões de representação. Com o apoio desta teoria é possível fazer o design de percursos de aprendizagem que sejam cognitivamente econômicos, o que está associado ao sucesso escolar. Salientamos aqui que a TRRS tem a resolução de problemas em comum com os Cenários para Investigação e com a Investigação em sala de aula, uma vez que segundo este autor $(2009,2013)$ "A distinção dos dois tipos de transformações, que são as conversões e os tratamentos, constitui uma ferramenta precisa e necessária para analisar a atividade de resolução de problemas em matemáticas" (DUVAL, 2009, p.11).

Uma sala de aula pode ser vista/pensada de várias formas. A organização de uma sala de aula pode ser arrumada em fila, círculo, grupos de 3 ou 4 
estudantes e de outras formas mais. Assim como as propostas também podem variar.

Ou seja, o professor pode:

- Apresentar questões do tipo exercícios dentro da tríade DefiniçãoExemplo-Exercícios, característica do ensino tradicional;

- Introduzir o tema a partir de um vídeo debate sobre o tema, ou ainda em grupo com tarefas abertas (tarefas com uma gama de respostas possíveis ou mesmo sem respostas conhecidas em cuja vivência;

- (Levar os estudantes a pesquisas não explicitadas no enunciado) ou fechadas (tarefas com resposta única e conhecida, normalmente tal resposta é disponibilizada o estudante em um gabarito ao final do livro/apostila/aula).

Entretanto, diversas pesquisas (D'AMORE, 2007; Ponte 1995; SKOVSMOSE 2000) tem questionado a eficácia da tríade Definição- exemplo, exercícios e tem trazido alternativas a esta proposta de ensino tão difundida em nossas salas de aula ainda hoje.

Apresentamos aqui os cenários de investigação de Skovsmose (2000) em que o mesmo propõe que ações tomadas em sala para a produção de significado

Segue abaixo uma tabela ilustrativa indicando os seis ambientes possíveis elencados por este autor a partir da conjugação entre as mencionadas referências e paradigmas:

\begin{tabular}{|l|c|c|}
\hline & $\begin{array}{c}\text { Paradigma do } \\
\text { exercício }\end{array}$ & Cenário para Investigação \\
\hline Referência à Matemática pura & $(1)$ & $(2)$ \\
\hline Referência à Semi realidade & $(3)$ & $(4)$ \\
\hline Referência ao Mundo real & $(5)$ & $(6)$ \\
\hline
\end{tabular}

Tabela 1: Ambientes de Aprendizagem

Fonte: (SKOVSMOSE, 2000, p.8)

Os ambientes 1 e 2 estão inseridos dentro da matemática pura: 
As tarefas no ambiente 1 são do tipo "resolva", "determine", ligue a coluna da esquerda à da direita". Questões do tipo "Calcule o valor de log100, Determine o valor de $\log _{24} 4$ ilustram esse cenário.

Já as tarefas no ambiente 2 são constituídas por questões abertas contextualizadas. $\mathrm{O}$ conjunto de tarefas que disponibilizamos no apêndice 1 deste guia ilustra este ambiente.

Apesar dos exercícios dos ambientes (1) e (2) estarem ligados à Matemática pura, é a forma com que se aborda e a maneira como se trabalha cada um é o que os diferencia.

Entendemos que o ambiente de aprendizagem (2), em que baseamos nosso trabalho, traz uma abordagem diferente para a abordagem da Matemática pura, propiciando ao aluno investigar sobre o assunto abordado uma vez que apresentamos um conjunto de situações problemas, sobre as quais os estudantes precisam comparar e identificar semelhanças e diferenças entre elas em busca de regularidades, de propriedades do conceito de logaritmos.

Neste ambiente não existe resposta única e às vezes é possível que não exista resposta, a importância está no percurso e não no destino. Não desenvolveremos neste guia os cenários 3,4,5 e 6 nos limitando a registrar a sua existência pois tal desenvolvimento foge ao escopo deste guia.

Já a investigação Matemática facilita a construção do raciocínio matemático dos estudantes uma vez que se constitui em momentos variados que os levam a: levantar questões, formular, testar e justificar conjecturas e a avaliar seu raciocínio. Sendo esperado destes que construam reflexões, segundo Ponte (1995):

A realização de tarefas exploratórias na conjunto de tarefas de Matemática são importantes porque elas: (a) constituem uma parte essencial da experiência Matemática e, por isso, permitem uma visão mais completa desta ciência; (b) estimulam 0 envolvimento dos estudantes, necessário a uma aprendizagem significativa; (c) podem ser trabalhadas por estudantes de ciclos diferentes, a níveis de desenvolvimento também diferentes; e (d) potenciam um modo de pensamento holístico (ao relacionarem muitos tópicos), essencial ao raciocínio matemático (CUNHA \& PONTE, 1995, p. 161). 
A investigação Matemática também se ocupa da resolução de problemas uma vez que segundo Ponte (2016) ${ }^{3}$ ambas pertencem à mesma categoria de prática de ensino.

$\mathrm{Na}$ minha perspectiva, as tarefas mais vocacionadas para servir de base a investigações Matemáticas podem ser consideradas como "problemas". Existem passagens de vários livros de Polya, por exemplo, em "Mathematical Discovery", em que são apresentadas e discutidas tarefas de investigação. Por isso, penso que se pode considerar a investigação na sala de conjunto de tarefas como uma forma de trabalho que se enquadra na resolução de problemas (PONTE, 2016, manuscrito).

O problema muito se diferencia do exercício uma vez que ele propicia o surgimento de reflexões na construção de conceitos matemáticos. Por problema entendemos que:

Tem-se, por outro lado, um problema quando uma, ou mais, das regras ou um, ou mais dos procedimentos necessários ainda não estão na bagagem cognitiva do responsável por resolvê-lo; na ocasião, algumas dessas regras ou algum desses procedimentos podem inclusive estar em via de explicitação; às vezes, é a própria sucessão de operações necessárias para resolver o problema que demandará um ato criativo por parte de quem precisa resolvê-lo (D'AMORE, 2007, p. 286).

Quanto aos exercícios sabemos que os mesmos possuem respostas únicas e, portanto, podem não levar os estudantes a reflexões aprofundadas. $O$ esquema abaixo explicita a diferença entre problema e exercício.

\begin{tabular}{|l|l|l|}
\hline & \multicolumn{1}{|c|}{ Problema } & \multicolumn{1}{c|}{ Exercício } \\
\hline No ensino & $\begin{array}{l}\text { Instrumento de aquisição de } \\
\text { conhecimento }\end{array}$ & $\begin{array}{l}\text { Instrumento para consolidar } \\
\text { conhecimentos e habilidades }\end{array}$ \\
\cline { 2 - 3 } & Objeto de ensino para inferir \\
\hline Privilegia & Processos & $\begin{array}{l}\text { Instrumento ponhecimentos e habilidades } \\
\text { condes }\end{array}$ \\
\hline O professor & Escolhe os problemas & Produtos \\
\cline { 2 - 3 } & Segue os processos & Escolhe os exercícios \\
\hline $\begin{array}{l}\text { O sujeito tem um } \\
\text { papel }\end{array}$ & Produtivo & Executivo \\
\hline
\end{tabular}

Tabela 2- Diferenças entre problemas e exercícios

${ }^{3}$ Notas de palestra. 
(DAMORE e ZAN apud D'AMORE, 2007, p. 300)

Como nos interessamos pela aprendizagem como processo, fizemos a opção pelo uso de problemas para o conjunto de tarefas que apresentamos neste guia.

\section{O QUE SÃO LOGARITMOS AFINAL?}

Logaritmos são expoentes e é nesta ideia que estão amarradas todas as atividades que elaboramos a partir de nossa pesquisa de mestrado. Estas atividades utilizam coordenação cognitivamente econômica de representações, como previsto pela TRRS, e buscam construir a compreensão da definição de logaritmos através do trabalho com propriedades da potenciação.

Encontramos em uma crônica de Carlos Heitor Cony ${ }^{4}$ uma tradução para o inconsciente coletivo que explicita a pouca familiaridade das pessoas em geral os logaritmos quando ele diz que,

Minhas relações com as Matemáticas nunca foram boas - e exagero ao falar em Matemáticas, no plural e na maiúscula. Nem mesmo a elementar aritmética privou de muita intimidade com meu impenetrável cérebro. Por todos os chamados bancos escolares que lustrei em minhas andanças, sempre deixei a merecida fama de refratário aos números, às operações, às frações e às regras de três. Não cito os logaritmos porque seria um escárnio de minha parte mencionar tais entidades[...] (CONY, 2005, p 13-14).

e que exemplifica algumas reações de nossos estudantes ao tema. O que nos levou a questionar se tal desconforto se manteria caso a relação logaritmo $=$ expoente fosse claramente delineada.

Briggs e Napier, no início do século XVII, observaram que era possível operar com os expoentes de potências. Ou seja, transforma multiplicações e divisões de potências em adições e subtrações de expoentes para encontrar o resultado da potência. Esta observação se constituiu em uma revolução tecnológica uma vez que esta descoberta permitiu que os cálculos dos grandes números com os quais os astrônomos lidam permitindo avanços inquestionáveis

${ }^{4}$ Carlos Heitor Cony é um cronista e escritor brasileiro, ocupa a cadeira de número 3 da Academia Brasileira de Letras. 
para a ciência ${ }^{5}$. O cerne do conjunto de tarefas elaborado reside, portanto, em uma abordagem na qual coordenamos, de forma facilitada, diferentes representações de um mesmo objeto matemático e no qual associamos diretamente o conceito de logaritmo com o conceito de potenciação.

\section{O CONJUNTO DE TAREFAS}

O conjunto de tarefas (tabela 3) resultante de nossa dissertação de mestrado que aqui apresentamos é composto de três momentos: 1) O primeiro dedicado a construir a motivação dos participantes em empreender uma investigação matemática, dele faz parte a tarefa 1 , o segundo dedicado à matemática em si, dele fazem parte as tarefas 2,3 e 4 e o terceiro dedicado à avaliação do conjunto de tarefas como um todo, dele faz parte a tarefa 5. A vivência do conjunto como um todo pode ser feita em 7 aulas. Para a aplicação do conjunto de tarefas propomos a divisão da turma em grupos de não mais do que 4 estudantes, pois grupos maiores facilitam a dispersão (KINDEL, 2013).

\begin{tabular}{|l|l|}
\hline \multicolumn{1}{|c|}{ Tarefa } & \multicolumn{1}{c|}{ Função } \\
\hline Caixas misteriosas & Disparadora \\
\hline Investigação de sequências & Investigação em uma perspectiva livre \\
\hline Coordenação de sequências & Exploração orientada \\
\hline Formalização da vivência & Sistematização em uma perspectiva diretiva \\
\hline Avaliação & $\begin{array}{l}\text { Avaliação das tarefas anteriores: Avaliação de } \\
\text { percurso e de conteúdo }\end{array}$ \\
\hline
\end{tabular}

Tabela 3: Tarefas do conjunto e suas funções

A primeira tarefa proposta é a investigação das caixas misteriosas cujo objetivo desta primeira tarefa é o de levar seus participantes a externar suas observações por meio da fala e da escrita, estimular suas inferências a partir do que individualmente ou em grupo é observado, despertar a motivação para a busca de consenso em torno de um problema apresentado, no caso o problema em questão é descobrir que objetos estão ocultos em cada caixa misteriosa analisada. Esta tarefa é realizada em 50 minutos, ou seja, uma aula.

\footnotetext{
${ }^{5}$ Mais informações em (IEZZI, 2010; LIMA, 1980; PAIVA, 2009).
} 
As caixas misteriosas consistem em seis caixas opacas lacradas, com estas em mãos vocês devem empreender uma investigação dividida em duas etapas, a primeira dedicada à observação, anotação e proposição de um palpite para o que está escondido no interior das caixas. O segundo se constitui em uma plenária no qual se busca o consenso entre os palpites de todos os grupos participantes, nesta seus grupos podem concordar uns com os outros e mudar seus palpites caso algum ou alguns outros grupos o convençam de seus argumentos. (Science Museum, 2016, tradução pessoal)

Ao percorrer de maneira informal as etapas do método científico, buscamos preparar o caminho para a investigação matemática visando o aprendizado deste conceito.

A tarefa 2 consiste na exploração de uma tabela contendo sequências numéricas diversificadas. O seu objetivo é levar os participantes a buscarem regularidades nas referidas sequências uma vez que estas se constituem em uma propriedade fundamental para a construção do conceito de logaritmo. A aplicação desta tarefa é feita em 100 minutos, ou seja, duas aulas.

Nesta tarefa é pedido aos participantes que observem a tabela 4 adiante e baseados nela respondam as perguntas 1 a 5 :

\begin{tabular}{|l|l|l|l|l|l|l|l|l|l|l|}
\hline & & & & & & & & & & \\
\hline a & 1 & 2 & 4 & 8 & 16 & 32 & 64 & 128 & 256 & 512 \\
\hline b & 1 & 5 & 25 & 125 & 625 & & & & & \\
\hline c & 0 & 4 & 8 & 12 & 16 & & & & & \\
\hline d & 1 & 3 & P & 27 & 81 & 243 & 729 & 2187 & 6561 & 19683 \\
\hline e & 0 & 0 & 0 & 0 & 0 & 0 & 0 & 0 & 0 & 0 \\
\hline f & 0 & 1 & 2 & 3 & 4 & 5 & 6 & 7 & 8 & 9 \\
\hline g & 0 & 3 & 6 & 9 & 12 & 15 & 18 & 21 & 24 & 27 \\
\hline h & 1 & 1 & 1 & 1 & 1 & 1 & 1 & 1 & 1 & 1 \\
\hline i & 1 & $1 / 3$ & $1 / 9$ & $1 / 27$ & $1 / 81$ & $1 / 243$ & $1 / 729$ & $1 / 2187$ & $1 / 6561$ & $1 / 19683$ \\
\hline J & 0 & -1 & -2 & -3 & -4 & -5 & -6 & -7 & -8 & -9 \\
\hline k & 1 & -2 & -4 & -8 & -16 & -32 & -64 & -128 & -256 & -512 \\
\hline l & 1 & 1 & 2 & 3 & 5 & 8 & 13 & 21 & 34 & 55 \\
\hline
\end{tabular}

Tabela 4: Sequências numéricas para exploração

A partir da tabela acima pedimos aos participantes que respondam às seguintes perguntas: 1) Comparem as linhas entre si, analisem cada uma delas e escrevam pelo menos cinco frases sobre o que observaram; 2) Completem as linhas das letras $b$ e $c$.; 3) $O$ que acontece entre os elementos de uma mesma 
sequência? Expliquem sua resposta. E em seguida, encontrem mais três elementos para cada uma delas; 4) Se quisermos continuar cada uma das linhas, acrescentando elementos à esquerda, como devemos proceder?; 5) Comparem todas as linhas com a sequência da letra f. O que observam?

$\mathrm{Na}$ tarefa 3, propomos aos participantes que relacionem sequências da tabela utilizada na tarefa anterior buscando regularidades entre elas. Nosso objetivo aqui é que os participantes percebam que um caminho para completar as sequências é a utilização de operações entre expoentes e também abrir caminho para o trabalho com o conceito de logaritmos abordando especificidades de sua definição ${ }^{6}$ sem, no entanto, abordá-la diretamente uma vez que o conjunto em questão visa trabalhar com a introdução a este conceito, esta tarefa é feita em 100 minutos, ou seja, duas aulas.

Nela pedimos aos participantes que observem a tabela 1 preenchida na atividade anterior $\mathrm{e}$ relacionem as linhas indicadas nos itens 1 a 7 desta escrevendo o que observam: 1)Linhas A e F; 2)Linhas D e F; 3) Linhas D e G; 4)Linhas H e F; 5) Linhas E e F; 6) Linhas I e J; 7) Existem outras duplas de linhas que vocês compararam? O que observam nelas?

$\mathrm{Na}$ tarefa 4 , os estudantes são convidados a formalizar as inferências levantadas nas tarefas 2 e 3 . Nosso objetivo aqui é identificar o lugar de um número em uma tabela, identificar cada uma das propriedades do logaritmo trabalhando a ideia de lugar na tabela e associar que o lugar de um número na tabela é o mesmo que encontrar o log b a. Esta tarefa é feita em 100 minutos, ou seja, duas aulas.

Nesta tarefa pedimos aos estudantes que, utilizando a tabela 8 abaixo, respondam às questões 1 e 2 :

\begin{tabular}{|l|l|l|l|l|l|l|l|l|l|l|l|}
\hline A & $-\mathbf{5}$ & $\mathbf{- 4}$ & $\mathbf{- 3}$ & $\mathbf{- 2}$ & $\mathbf{- 1}$ & $\mathbf{0}$ & $\mathbf{1}$ & $\mathbf{2}$ & $\mathbf{3}$ & $\mathbf{4}$ & $\mathbf{5}$ \\
\hline B & $1 / 32$ & $1 / 16$ & $1 / 8$ & $1 / 4$ & $1 / 2$ & 1 & 2 & 4 & 8 & 32 & 64 \\
\hline C & $1 / 243$ & $1 / 81$ & $1 / 27$ & $1 / 9$ & $1 / 3$ & 1 & 3 & 9 & 27 & 81 & 243 \\
\hline
\end{tabular}

${ }^{6}$ Um logaritmo pode ser definido por $\mathrm{a}^{\mathrm{x}}=\mathrm{b}$, isso implica em que $\mathrm{x}=\log _{\mathrm{a}} \mathrm{b}$, sendo que $\mathrm{b}$ e a são maiores que zero e a é diferente de um e maior que zero. Temos que a é a base do logaritmo, b é o logaritmando ou antilogaritmo e x é o logaritmo (IEZZI et al 2010; PAIVA, 2009). 
http://dx.doi.org/10.5965/2357724X06112018377

\begin{tabular}{|l|l|l|l|l|l|l|l|l|l|l|l|}
\hline $\mathbf{D}$ & $1 / 1024$ & $1 / 256$ & $1 / 64$ & $1 / 16$ & $1 / 4$ & 1 & 4 & 16 & 64 & 256 & 1024 \\
\hline E & $1 / 3125$ & $1 / 625$ & $1 / 125$ & $1 / 25$ & $1 / 5$ & 1 & 5 & 25 & 125 & 625 & 3125 \\
\hline $\mathbf{F}$ & $1 / 100000$ & $1 / 10000$ & $1 / 1000$ & $1 / 100$ & $1 / 10$ & 1 & 10 & 100 & 1000 & 10000 & 100000 \\
\hline
\end{tabular}

Tabela 5: potências diversas

Questão 1:Se estabelecemos, utilizando a linha A como referência, o lugar que 32 ocupa na sequência de 2 temos a posição 5 . Formalizando esta frase podemos substituí-la por $\log _{2} 32$. Utilizando o enunciado encontre o lugar de:

a) $\log _{2} 64=(O$ lugar que 64 ocupa na sequência de 2)

b) $\log _{381} 81$ (O lugar que 81 ocupa na sequência de 3)

c) $\log _{4} 1 / 16=(O$ lugar que $1 / 16$ ocupa na sequência de 4)
d) $\log _{5} 125=$
f) $\log _{4} 256=$
h) $\log 101000=$
e) $\log 1 / 1000=$
g) $\log _{2} 256=$

${ }^{*} \log _{10} 100=\log 100$ (Toda vez que nos referimos ao lugar de um número na sequência de 10, o registro fica simplificado como log do número sem escrever a base, Istoé, log 100).

Questão 2: É possível encontrar o lugar de um logaritmo de maneira indireta. Aqui propomos 4 maneiras, utilizando cada uma delas responda às letras $a, b$ e $c$ utilizando cada um dos itens abaixo como modelo:

i) $\log _{2} 32=\log _{2} 4.8=2+3=5$, ou seja, $\log a \cdot b=\log a+\log b$
a) $\log _{4} 64=$
b) $\log _{2} 64=$
c) $\log _{2} 1024=$

ii) $\log _{2} 8=\log _{2} 32: 4=5-2=3$, ou seja, $\log a: b=\log a-\log b$
a) $\log _{5} 1 / 125=$
b) $\log _{5} 1 / 625=$
c) $\log _{3} 9=$

iii) $\log _{2} 4^{2}=2 \cdot \log _{2} 4=2.2=2^{2}=4$, ou seja, $\log a^{b}=b \cdot \log a$
a) $\log _{2} 4^{2=}$
b) $\log _{4} 4^{2}=$
c) $\log _{3} 3^{2}=$
iv) $\log _{4} 16=\log _{2} 16 / \log _{2} 2=4 / 1=4$, ou seja, $\log _{b} a=\left(\log _{c} a\right) /\left(\log _{c} b\right)$
a) $\log _{2} 32=$
b) $\log _{2} 64=$
c) $\log _{3} 81=$ 
Para o fechamento deste conjunto de tarefas propomos duas avaliações com duração de 15 minutos cada, opcionais, para as tarefas anteriores: 1)avaliação do percurso e 2) Avaliação conceitual:

1) $\mathrm{Na}$ avaliação do percurso, o objetivo é o de coletar as impressões e sugestões dos participantes para a melhoria do conjunto. Uma sugestão para este fechamento é a aplicação do questionário que elaboramos composto da tabela 6

\begin{tabular}{|l|l|l|l|l|l|}
\hline & Ótimo & Bom & Regular & Ruim & Péssimo \\
\hline Conteúdo: na & & & & & \\
\hline $\begin{array}{l}\text { Clareza } \\
\text { apresentação }\end{array}$ & & & & & \\
\hline $\begin{array}{l}\text { Materiais } \\
\text { disponibilizados }\end{array}$ & & & & & \\
\hline Duração & & & & & \\
\hline Ambiente & & & & & \\
\hline
\end{tabular}

Tabela 6: Parte objetiva do questionário

e das perguntas adiante:

a. Qual atividade mais gostou e por que?

b. O que você achou da matemática apresentada desta forma?

c. Na sua opinião quão é o ponto positivo? E o negativo?

d. Avalie, de forma individual, o conjunto de tarefas vivenciado.

2) $\mathrm{Na}$ avaliação conceitual o objetivo é o de reunir as inferências dos estudantes em torno da definição e propriedade dos logaritmos.

Propomos aqui que os alunos respondam, com suas próprias palavras às perguntas sugeridas.

a) O logaritmo pode ser negativo, zero ou um? Por quê?

b) A base de um logaritmo pode ser negativa, zero ou um? Por quê? 
c) O que é o logaritmo na sua opinião?

Nosso intuito foi o de oferecer, a docentes interessados no tema, um percurso fundamentado teoricamente para o ensino aprendizagem de logaritmos. Neste guia, visando o dia a dia corrido dos docentes em geral. Oferecemos também, com o objetivo de facilitar o trabalho em sala de aula, o conjunto de atividades proposto já pronto para impressão. Com a fundamentação teórica as tarefas e as avaliações disponibilizadas aqui neste guia esperamos auxiliar professores a construir com seus educandos o conceito de logaritmo como expoente, através da exploração de suas propriedades e da reflexão sobre as mesmas em um ambiente investigativo.

\section{REFERÊNCIAS BIBLIOGRÁFICAS}

BATISTA, Adriana Maria da Silva Barbosa; SPINILLO, Alina Galvão. Nem todo material concreto é igual: a importância dos referentes na resolução de problemas. Estud. psicol. (Natal), Natal, v. 13, n. 1, p. 13-21, Abr. 2008.

CONY, C.H. CéstlaGuerre!". Antologia de Crônicas, org. Herberto Sales, $3^{\mathrm{a}}$ ed., São Paulo: Ediouro, 2005, p. 13-14

CUNHA, H. OLIVEIRA, H. PONTE, J.P. Investigações Matemáticas na sala de conjunto de tarefas. Actas do ProfMat95, Lisboa: APM, 1995 (p. 161-167)

IEZZI et al. Matemática Ciência e Aplicações. 6 ed. São Paulo: Saraiva, 2010.

D'AMORE, B. Elementos de didática da Matemática. Tradução de Maria Cristina Bonomi Barufi. São Paulo: Livraria da Física Editora, 2007.

IGLIORI, S.B.C. MARANHÃO,M.C. Registros de Representação e Números Racionais do livro: Aprendizagem de Matemática. In: Silvia Dias Alcântara Machado (org) Aprendizagem de Matemática - Registros de Representação Semiótica: Campinas SP: Papirus, 2013.p. 57-70.

KINDEL, D.S. Investigações em Sala de Conjunto de tarefas de Matemática: a Geometria Fractal e as Sequências Numéricas Infinitas Xi Encontro Nacional de Educação Matemática Curitiba - Paraná, 20 a 23 de julho de 2013 Anais do XI Encontro Nacional de Educação Matemática - ISSN 2178-034X.

LIMA, E.L. Logaritmos. Rio de Janeiro: SBM, 1980. 
LIMA, F. Dica saudável, bolor no laboratório de Matemática. Revista Cálculo, São Paulo, n. 49, p. 21- 25, fev. 2014.

NAVAS, M.G.M. Medidas em telecomunicações e eletrônica, Ed. SENAC, Rio de Janeiro, 2015.

PONTE, J.P.; BROCADO, J.; OLIVEIRA, Investigações Matemáticas em Sala de Conjunto de tarefas. Autêntica. Belo Horizonte. 2005.

SKOVSMOSE, O. Cenários para investigação. Bolema. Ano 13, n. 14, 2000. p. 66 a 91.

. Educação Crítica: Incerteza, Matemática, Responsabilidade, tradução de Maria Aparecida Viggiani Bicudo, Cortez Editora, São Paulo, 2007.

SILVA. S. F.; NÚÑEZ. I. B.. O Ensino por problemas e trabalho experimental dos estudantes - reflexões teórico-metodológicas. Revista Química Nova, v.25, n.6b, p.1197-1203, dez. 2002. Disponível em: < http://www.scielo.br/scielo. php?script=sci_arttext\&pid=S0100- 40422002000700023.

SILVA, Maria Regina Gomes da. Avaliação: um contrato de trabalho. Interface (Botucatu), Botucatu, v. 2, n. 2, p. 155-172, Feb. 1998 . Disponível em <http://www.scielosp.org/scielo.php?script=sci_arttext\&pid=S1414$32831998000100009 \& \operatorname{lng}=e n \& n r m=i s o>$. Acesso em abr. 2016

SIMÕES, M., SÔNEGO, D. A coisa sem sentido faz sentido há séculos. Revista Cálculo, São Paulo. Fascículo 33, p.43-54. Out, 2013. 